\title{
The sporadic radiant and distribution of meteors in the atmosphere as observed by VHF radar at Arctic, Antarctic and equatorial latitudes
}

\author{
P. T. Younger, I. Astin, D. J. Sandford, and N. J. Mitchell \\ Centre for Space Atmospheric and Oceanic Science, Department of Electronic \& Electrical Engineering, University of Bath, \\ Claverton Down, Bath, BA2 7AY, UK
}

Received: 7 November 2008 - Revised: 5 June 2009 - Accepted: 3 July 2009 - Published: 15 July 2009

\begin{abstract}
Results are presented of a study of the temporal and spatial variability in meteor count rate observations from three VHF meteor radars. These radar are located in the Arctic (at Esrange, $68^{\circ} \mathrm{N}$ ), in the Antarctic (at Rothera, $68^{\circ} \mathrm{S}$ ) and near the Equator (on Ascension Island, $8^{\circ} \mathrm{S}$ ). It is found that for all three locations there is a strong diurnal cycle in observed hourly meteor counts and the time of maxima and minima in these counts depends on the month of the year. In addition, at high latitude there is a strong annual cycle in observed monthly-mean meteor counts, whereas for the radar at low latitude there is a semi-annual cycle. At high latitude there is also an annual cycle in the mean height at which meteors are observed. However, no such annual cycle is found in observed meteor count rates from the low latitude radar. The meteor count data from all the radars are combined to investigate the sporadic radiant distribution (i.e. the distribution of direction of arrival on the celestial sphere of sporadic meteors). This combined radiant distribution shows that there are six main source regions for meteors. The latitudinal and temporal dependence in observed meteor count rates appears to result from a combination of the sporadic radiant distribution, annual fluctuations in atmospheric density, the sensitivity of the radar to meteors from different source directions and the temporal and spatial variability in meteor fluxes.
\end{abstract}

Keywords. Interplanetary physics (Interplanetary dust; General or miscellaneous) - Radio science (Remote sensing)

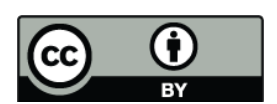

Correspondence to: I. Astin (eesia@bath.ac.uk)

\section{Introduction}

Meteors are the visible manifestation of meteoroids (extraterrestrial dust) as they ablate on entering the Earth's atmosphere. They are classified as being either part of a shower, or part of the background "sporadic" meteor flux. This study concentrates on the latter (sporadic meteors) which contribute the overwhelming majority of meteoroid material entering the atmosphere (e.g. Ceplecha et al., 1998; Baggaley, 2002). The deposition of this material is neither isotropic in direction nor constant in time. For example, studies such as Campbell-Brown and Jones (2006) show that the sporadic meteors appear to originate primarily from six rather diffuse sources. Two of these, the prograde and retrograde apex sources, lie near the point on the celestial sphere towards which the Earth appears to be moving (Galligan and Baggaley, 2006). The four other sources are the helion (sunward) source, the anti-helion source and two sources at high ecliptic latitudes known as the north and south toroidal sources (Hawkins, 1956; Jones and Brown, 1993). The positions of these sources appear to result from the Earth's motion around the Sun and the distribution of the orbits of meteoroids about the Sun.

In addition to this spatial anisotropy, temporal variations have also been observed in the deposition of meteoroid material into the atmosphere. Such variations have been attributed to (1) fluctuations in the number density of meteoroids in space, (2) geometrical factors relating to the location of observing sites and position of the sources, and (3) seasonal changes in the atmosphere that could effect the observation of meteors (e.g. Singer et al., 2004; Campbell-Brown and Jones, 2006).

In the work reported here the latitudinal, seasonal, temporal and spatial variability in observed meteor count rate observations from three VHF meteor radars is described. These

Published by Copernicus Publications on behalf of the European Geosciences Union. 
(a)

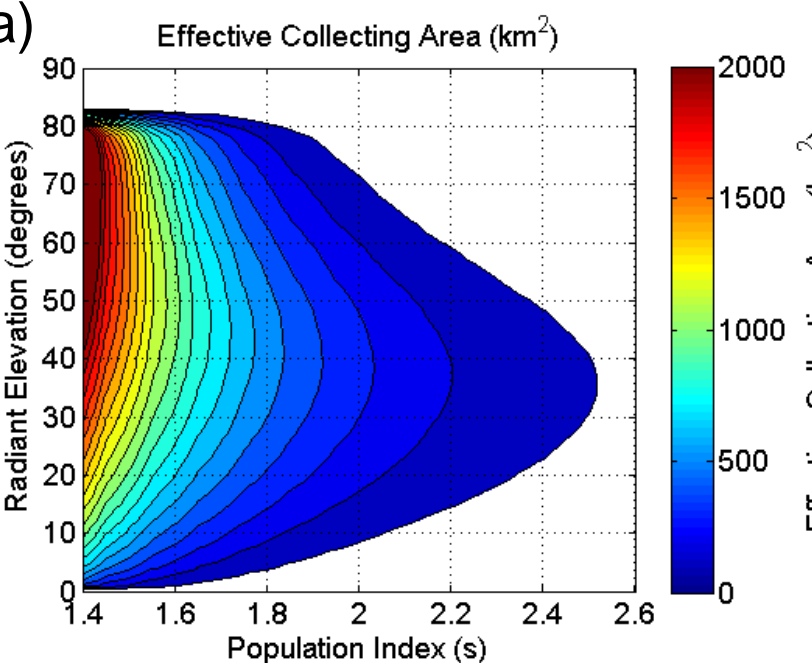

(c)

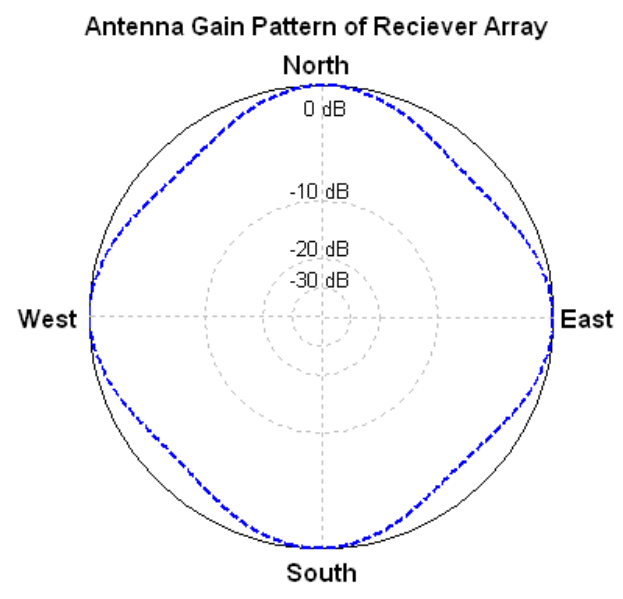

(d) (b)

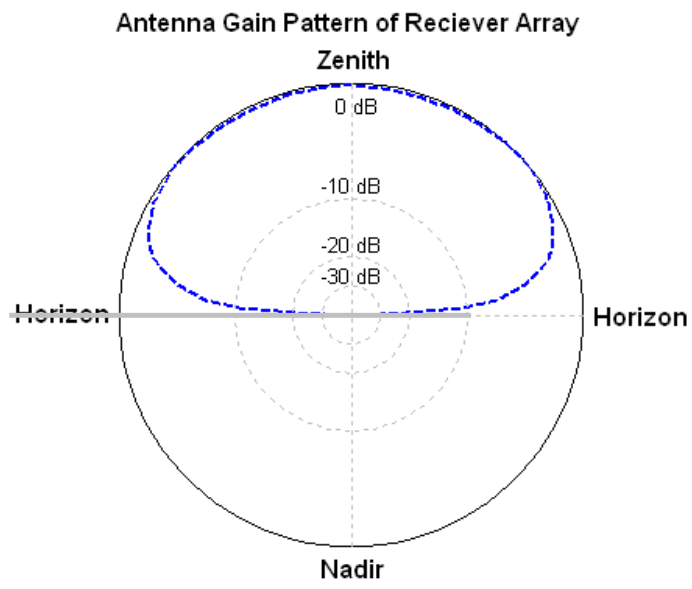

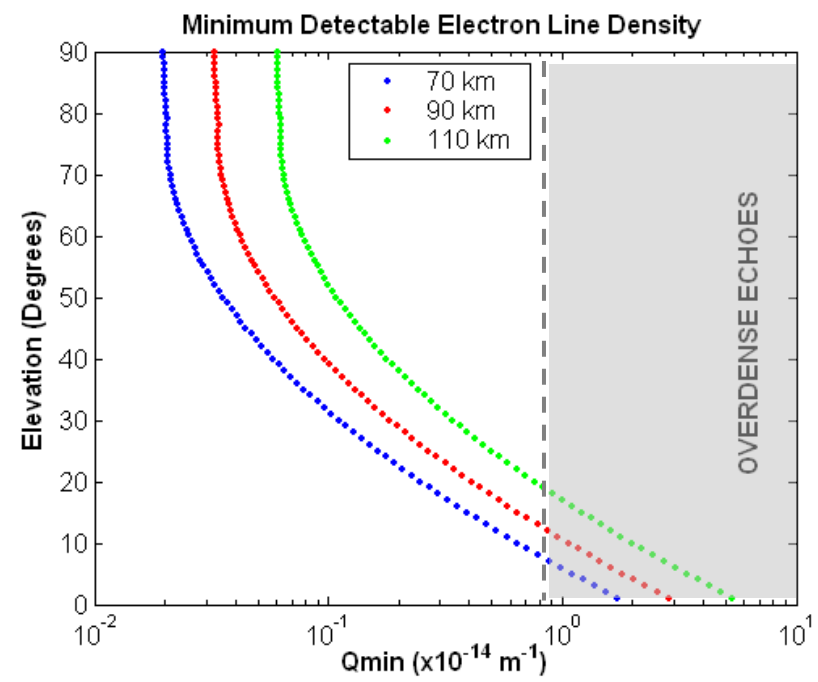

Fig. 1. (a) The Effective radar collecting radar area as a function of source elevation for the Esrange radar. (b) The polar diagram with respect to elevation, and (c) the polar diagram with respect to azimuth, of a receiving antenna used at Esrange, Ascension Island and Rothera. (d) The minimum detectable electron line density as a function of elevation for a $6 \mathrm{~kW}$ system for meteor velocities of $25 \mathrm{~km} \mathrm{~s}^{-1}$ at heights of $70 \mathrm{~km}$ (blue dots), $90 \mathrm{~km}$ (red dots) and $110 \mathrm{~km}$ (green dots).

meteor count rates are used to investigate the sporadic radiant distribution. The latitudinal, seasonal, temporal and spatial variability is explained in terms of this distribution, annual fluctuations in atmospheric density, sensitivity of the radar to meteors from different source directions and diurnal and height variations in meteor fluxes.

\section{Data and analysis}

The instruments used in this study are meteor radars located at Esrange $\left(68^{\circ} \mathrm{N}, 21^{\circ} \mathrm{E}\right)$, Ascension Island $\left(8^{\circ} \mathrm{S}, 14^{\circ} \mathrm{W}\right)$ and Rothera $\left(68^{\circ} \mathrm{S}, 68^{\circ} \mathrm{W}\right)$. Their main features are summarised in Table 1. All three are commercially produced Skiymet "all-sky" VHF systems optimised for measurements of upper atmospheric winds (see Hocking et al., 2001, for a detailed description of the radars). Each consists of a single Yagi transmitting antenna and a 5-element receiving interferometer. The radars have a pulse width of $13 \mu$ s and a pulse repetition frequency of $2144 \mathrm{~Hz}$.

The radars operate by detecting the drift of the ionisation trail produced during the ablation of a meteoroid. However, each radar can only observe this meteor trail if the trail is perpendicular to the radar beam (resulting in specular 
Table 1. Main features of the three meteor radars used in this study.

\begin{tabular}{llll}
\hline & Esrange & Ascension Island & Rothera \\
\hline Frequency & $32.5 \mathrm{MHz}$ & $43.5 \mathrm{MHz}$ & $32.5 \mathrm{MHz}$ \\
Peak power & $6 \mathrm{~kW}$ & $12 \mathrm{~kW}(6 \mathrm{~kW}$ after October 2005) & $6 \mathrm{~kW}$ \\
Observational period & $1999-2008$ & $2001-2007$ & $2005-2008$ \\
\hline
\end{tabular}

reflection). This reduces the number of meteors observed as compared to their total number. In addition, the detection system used in the Skiymet rejects "overdense" meteor echoes (that is, where the local electron density, produced during ablation, is so high that the electron plasma frequency in the trail exceeds the radar frequency). This rejection of overdense meteors prevents multiple recording of the same meteor.

To better quantify the sensitivity of the radar to a meteor from a source at a given azimuth and elevation we use the method of Brown and Jones (1995) to define an effective "collecting area". For brevity we refer the reader to that study for further details. This area is a function of both radar parameters and meteoroid mass distribution. The latter, an observed power-law distribution $\left(d N \propto m^{-s} d m\right)$ is defined by a parameter called the mass index $s$. In this, a smaller value of $s$ implies a greater number, $N$, of meteoroids with larger mass, $m$. Studies suggest that $s$ lies within the range 1.8 to 2.4 for sporadic meteors (e.g. Simek and McIntosh, 1968).

Figure 1a shows the effective collecting area of the Esrange radar (for $s=1.8, s=2.0$ and $s=2.2$ ) as a function of elevation angle. This is typical of all three radars used in this study. We see that the effective collecting area increases quickly with decreasing $\mathrm{s}$ and that the radar is most sensitive to sources at elevation angles between $40^{\circ}-50^{\circ}$ for middle values of $s$. In contrast, the antenna gain (Fig. $1 \mathrm{~b}$ and c) and associated sensitivity of the radar are largely independent of azimuth and zenith.

The radar detection is dependent on the number of electrons produced per metre travelled by a meteor as it ablates. This is known as the electron line density. The minimum electron line density that can be detected by the Esrange radar as a function of elevation and height is given in Fig. 1d. Similar results are found for the two other radars. The minimum detectable electron line density increases with height due to the increase in initial radius of the meteor trail with height. Hence, the radars are less sensitive to meteors at greater heights.

In addition to the effective collecting area several other factors affect the meteor count rate. These generally reduce the observed meteor count rate. These include the finite velocity effect and the pulse repetition factor. The finite velocity effect is where the meteor trail forms in a region of high diffusion rate and so will change significantly within the first Fresnel zone of the radar. This reduces the scattering cross- section of the trail. As a result, it is possible for an otherwise detectable meteor to fall below the detection threshold of the radar. This bias was corrected for in our radars by determining the "attenuation factor" using the method of Cervera and Elford (2004). The pulse repetition rate factor comes into play when the decay time of a meteor echo is smaller than the inter-pulse period of the radar. In this case it is possible for a trail to form and decay between radar pulses. This means that the meteor is not observed by the radar. For the radars used in this study the inter-pulse period is $0.5 \mathrm{~ms}$ and hence for our radars this effect is very small. Even so, this effect was corrected for by determining the "echo rate reduction factor" following Ceplecha et al. (1998) and Galligan and Baggaley (2004).

In addition, meteors ablating at greater heights generate larger initial meteor trail radii than those at lower heights. This affects the Ascension Island radar more than the other two radars, because of its higher frequency. At these larger initial trail radii, destructive interference within the trail results in attenuation of the radar echo power. This effect is most prevalent at heights over $100 \mathrm{~km}$. This is corrected for following the experimental work of Jones and CampbellBrown (2005) again by assigning an attenuation factor.

Ellyett and Kennewell (1980), using the earlier theories of Kaiser and Closs (1952) and Kaiser (1960, 1961), contend that changes in atmospheric scale height (and hence density and density gradients) can produce significant variations in the observed meteor echo count rate. These not only affect the heights at which meteoriods ablate, but also the area over which a meteoroid will ablate. This implies that when the atmospheric scale height is small the meteor will ablate more quickly and over a shorter path than for a larger scale height. Thus, the electron line density will be greatest when the atmospheric scale height is at a minimum. Therefore, when the scale height is small, some meteoroids that would normally be below the detection threshold of the radar will be detected and hence count rates will increase accordingly. This implies that there should be a seasonal variation in meteor counts solely resulting from changes in the atmosphere. 
(a)
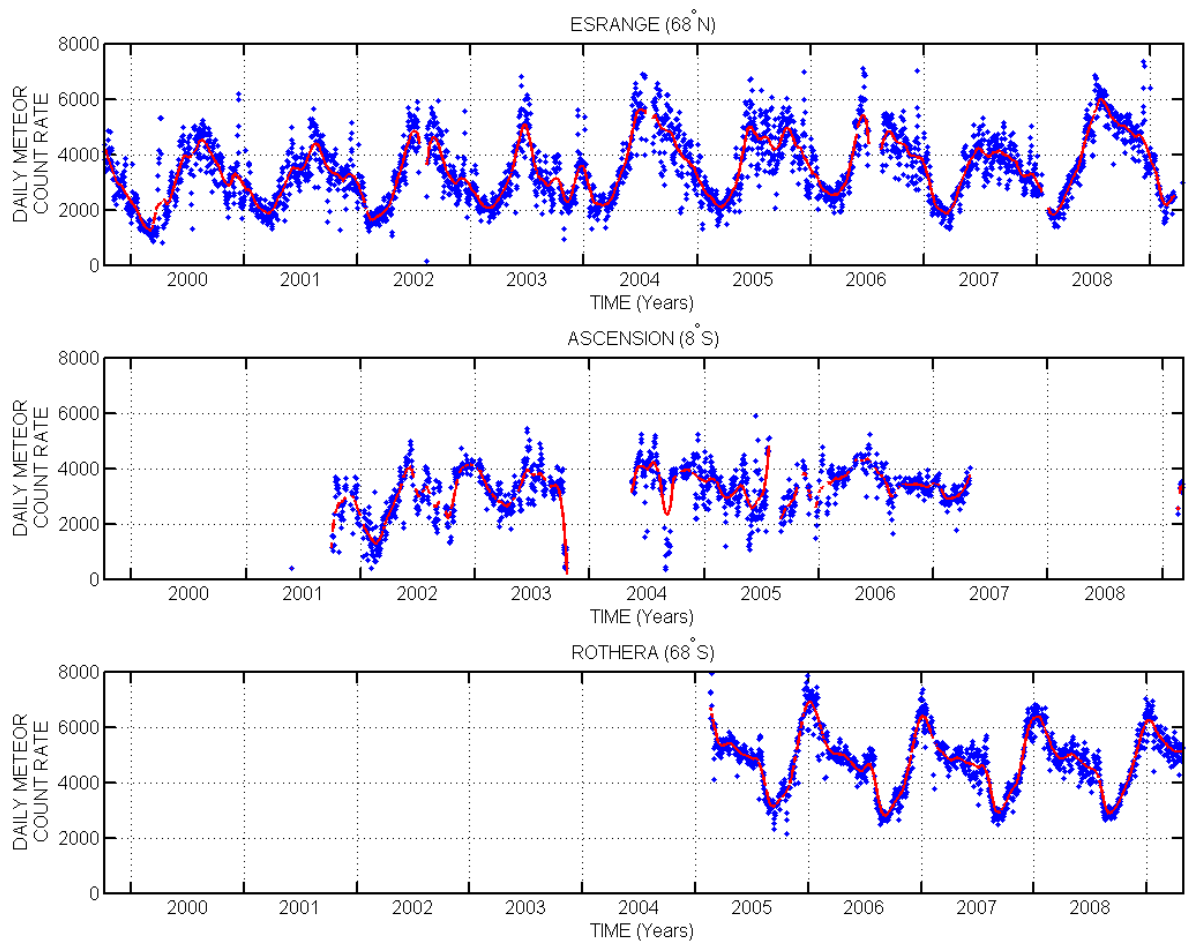

(b)
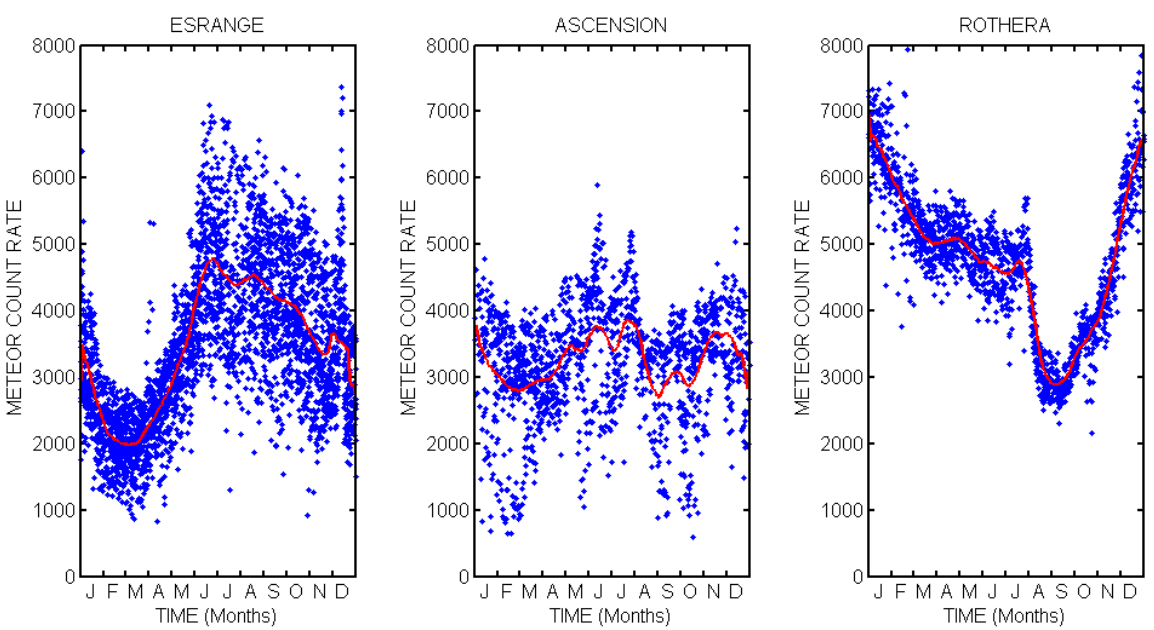

Fig. 2. (a) Daily meteor count rate (blue dots) at Esrange (1999-2008), Ascension Island (2001-2007), and Rothera (2005-2008). The red line is the 30-day running mean. (b) Composite daily meteor count rate (blue dots) versus calendar date. Red line is the 30-day running mean.

\section{Results}

\subsection{Observed meteor count rates}

Daily meteor count rates observed at Esrange $\left(68^{\circ} \mathrm{N}\right)$, Ascension Island $\left(8^{\circ} \mathrm{S}\right)$ and Rothera $\left(68^{\circ} \mathrm{S}\right)$ are shown in Fig. 2. A clear annual cycle is apparent in the data from Esrange, with maximum count rates being observed in June followed by a slow decline in count rates throughout the period July to December. Minimum count rates of approximately $40 \%$ of maximum are detected in February and March. This minimum is followed by a rapid rise in count rate in the interval April to May. These results are very similar to those of Singer et al. (2004), using a meteor radar situated at the ALOMAR observatory $\left(69^{\circ} \mathrm{N}\right)$, who also found a strong 
annual variation in count rate, with maximum numbers being observed in June and minimum numbers in February.

Figure 2 shows that there is also an annual cycle in observed meteor count rate in the Antarctic data (from Rothera) where maximum count rates are observed in the austral summer (i.e. in December and January). This is followed by a slow decline in count rate throughout the period January to July, with minimum count rates (of approximately $50 \%$ of maximum) observed in September. This minimum is followed by a rapid rise in count rate in October and November. In contrast, over Ascension Island (in the equatorial region) the seasonal behaviour in meteor count rates follows a semiannual cycle, with maximum count rates observed near the summer and winter solstices, and minimum count rates, of approximately $70 \%$ of the maximum, observed in equinoctial months.

The average monthly-mean count rates of Fig. 2 conceal considerable variability over both the short-term and between different years. As an example, at Esrange a very clear annual cycle is observed. However, the "profile" of this annual cycle changes from year to year. This is most evident in comparing the duration of the summertime increase in meteor activity (for example, this is much shorter in 2003 than in 2004). In addition to the inter-annual and seasonal variability there is a strong variation in meteor count rate with time of day. The diurnal variation of observed meteor count rate per hour versus time (LT) for the three radars (Esrange, Ascension Island and Rothera) is displayed in Fig. 3a, b and c. At Esrange (Fig. 3a) the time of day of maximum meteor count rate has an annual cycle with maximum meteor count rates being observed at $\sim 07: 00 \mathrm{UT}$ (08:00 LT) in June. The time of maximum count rate occurs progressively earlier in the day throughout the interval July to January and in the period February to May occurs progressively later. The time of minimum meteor count rate also follows a similar seasonal trend, but lags the time of maximum count rate by $\sim 12 \mathrm{~h}$.

At Ascension Island (Fig. 3b) the time of maximum meteor count rate also follows a semi-annual cycle. Maximum meteor count rates were observed at $\sim 08: 00$ UT $(07: 00 \mathrm{LT})$ in January and February. It is interesting to note that at Ascension Island the time of minimum meteor count rate is stable throughout the year $(\sim 18: 00 \mathrm{UT})$.

At Rothera (Fig. 3c) the time of maximum meteor count rate also has a clear annual cycle with maximum meteor count rates being observed at $\sim 12: 00 \mathrm{UT}(08: 00 \mathrm{LT})$ in the interval December to April. The time of maximum count rate occurs progressively earlier in the day throughout the interval May to July until in July and August maximum count rates are observed at $\sim 10: 00 \mathrm{UT}$ (05:00 LT) and in the period September to November the time of maximum count rate occurs progressively later in the day. The time of minimum meteor count rate follows a similar seasonal trend, lagging the time of maximum count rate by $\sim 12 \mathrm{~h}$.

The radars also determine the height of each meteor echo. From these observations, monthly-mean height profiles over (a)

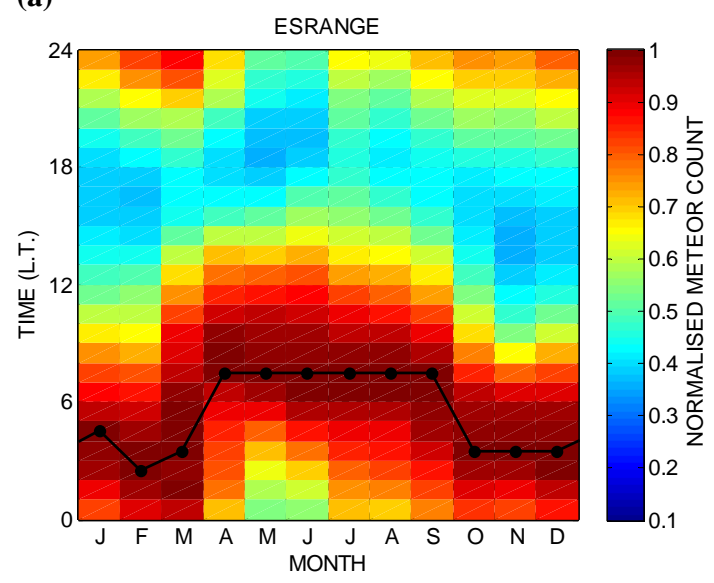

(b)

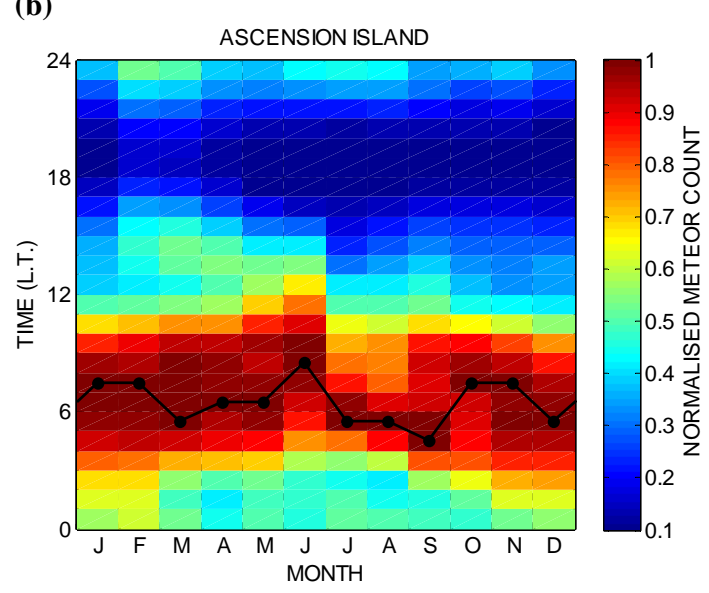

(c)

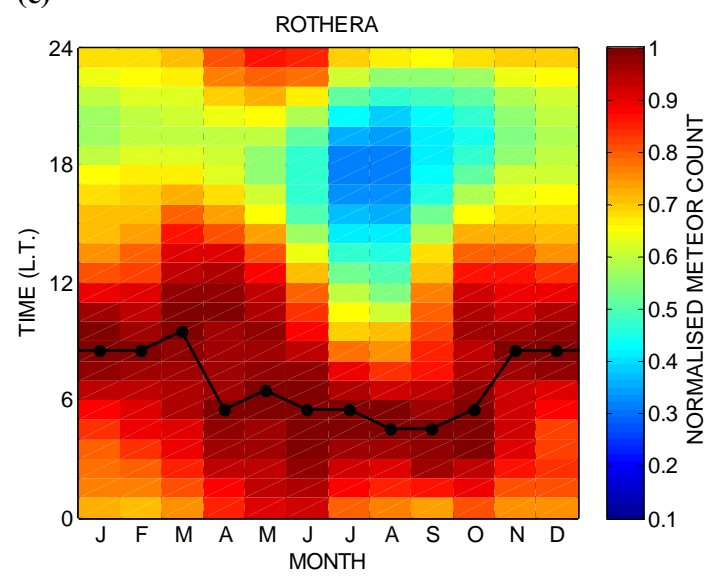

Fig. 3. Contour plots showing diurnal variation of hourly meteor count rates (in local time) at (a) Esrange (1999-2008), (b) Ascension Island (2001-2007), and (c) Rothera (2005-2008). Note the annual variations in meteor count rates.

the altitude range 70 to $110 \mathrm{~km}$ are derived. These, for Esrange $\left(68^{\circ} \mathrm{N}\right)$, Ascension Island $\left(8^{\circ} \mathrm{S}\right)$ and Rothera $\left(68^{\circ} \mathrm{S}\right)$, are shown in Fig. $4 \mathrm{a}, \mathrm{b}$ and c, respectively, with the mean height in each month plotted as a black circle. In Fig. 4a 


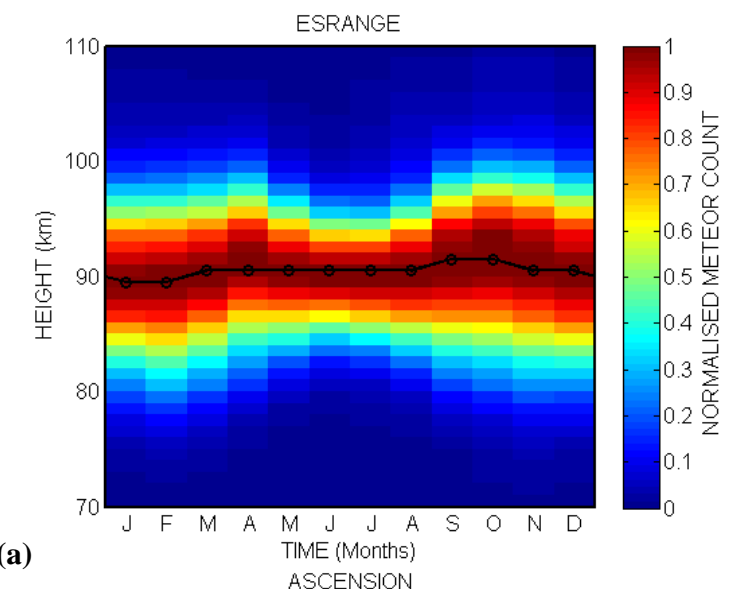

is seen in the summer months (June to August). An annual cycle in the height of peak meteor count rates is also observed at Rothera (Fig. 4c). During the period June to August (austral winter) peak count rates are detected at heights $\sim 88$ to $89 \mathrm{~km}$, whilst, during the summer/autumnal months (December to April) peak count rates are observed at heights $\sim 91 \mathrm{~km}$. These heights are similar to the results from Esrange. However, over Ascension Island (Fig. 4b) there is no significant seasonal change in the height of peak meteor count rates, which occurs at $\sim 90 \mathrm{~km}$ throughout the year.

The simultaneous measurements of Fig. 4a and c show a shift of approximately six months between the height variations in the Northern and Southern Hemispheres. As the Esrange and Rothera radars are identical instruments sited at conjugate latitudes this implies that the variation is likely atmospheric in origin (Ellyett and Kennewell, 1980) rather than a shift in meteor distribution which would produce a simultaneous shift in the height profile of meteor echoes in both hemispheres (McKinley, 1961). This is discussed further in Sect. 4.

\subsection{The sporadic radiant distribution}

Figure 5 presents the monthly-mean sporadic meteor radiant distributions of all meteors detected over Ascension Island for each month from January 2005 to December 2006 inclusive. These are derived using geocentric velocity of meteors versus heliocentric longitude and ecliptic latitude based on observations given in Galligan and Baggaley (2005).

The radiant distributions are plotted in the standard ecliptic coordinate frame where the centre of the plots gives the direction of the Earth's motion about the Sun. In this frame the Sun is always at $90^{\circ}$ to the left of this point. One of the first things to note about Fig. 5 is that all six sporadic sources identified by Galligan and Baggaley (2005) are represented. The helion and anti-helion sources (located approximately $70^{\circ}$ to the left and right of the direction of the Earth's motion) are meteoroids in highly elliptical low inclination orbits (Taylor and Elford, 1998) that strike the Earth postperihelion (in the case of helion source) or pre-perihelion (anti-helion source). In addition to these, the apex sources lie approximately $15^{\circ}$ above and below the ecliptic plane. The more northern of these appears to have a greater intensity than the southern one, and these sources appear weaker than the helion and anti-helion sources. This is in broad agreement with the studies of Taylor and Elford (1998) and Galligan and Baggaley (2005). Additionally, two weaker sources are observed to lie approximately $60^{\circ}$ above and below the ecliptic plane. These represent populations of meteoroids in high inclination orbits. The earlier work of Taylor and Elford (1998) also identify two sources but they place these toroidal sources at higher latitudes than presented here. This is possibly due to differences in techniques used to define the source regions. 


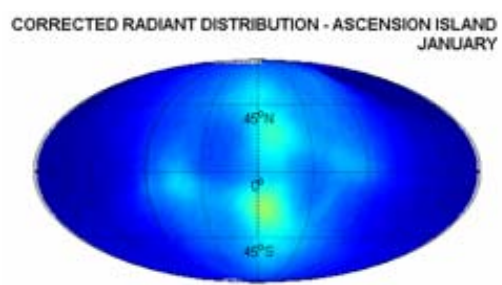

CORRECTED RADLANT DISTRIBUTION - ASCENSION ISLAND

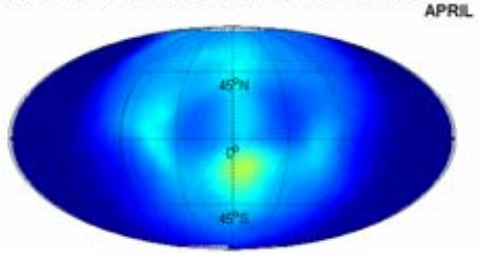

CORRECTED RADLANT DISTRIBUTION - ASCENSION ISLAND
JULY

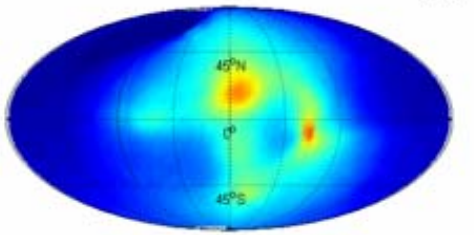

CORRECTED RADUNT DISTRIBUTION - ASCENSION ISLAND

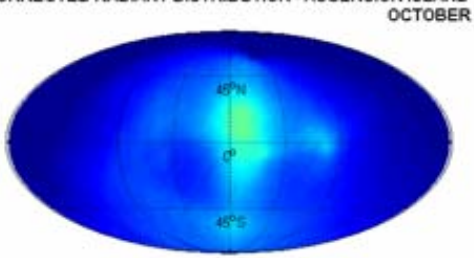

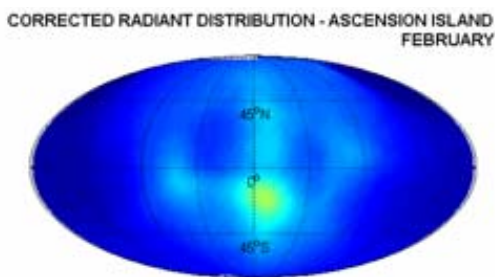

CORRECTED RADUANT DISTRIBUTION - ASCENSION ISLAND

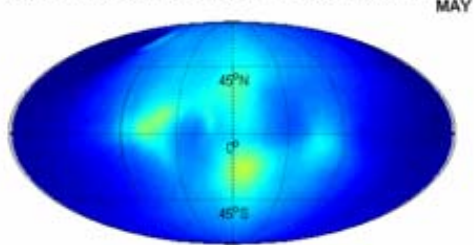

CORRECTED RADUANT DISTRIBUTION - ASCENSION ISLAND

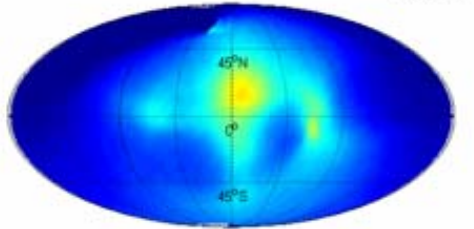

CORRECTED RADUANT DISTRIBUTION - ASCENSION ISLAND

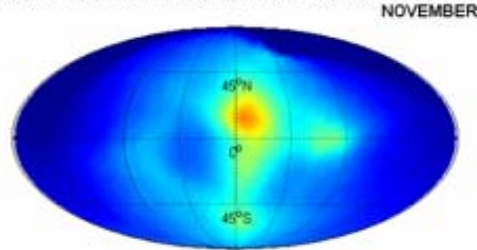

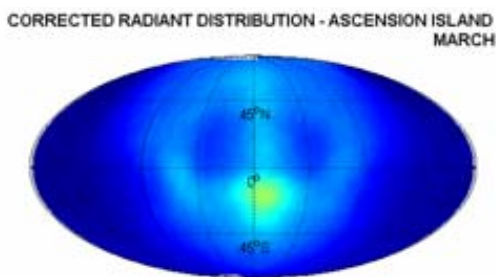

CORRECTED RADUANT DISTRIBUTION - ASCENSION ISLAND

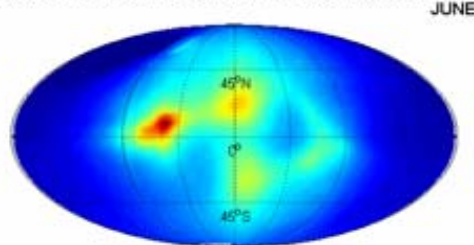

CORRECTED RADLANT DISTRIBUTION - ASCENSION ISLAND SEPTEMBER

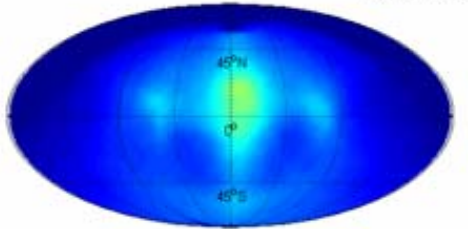

CORRECTED RADUNT DISTRIBUTION - ASCENSION ISLAND

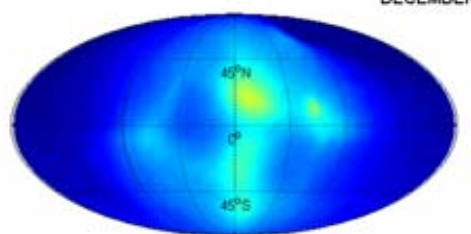

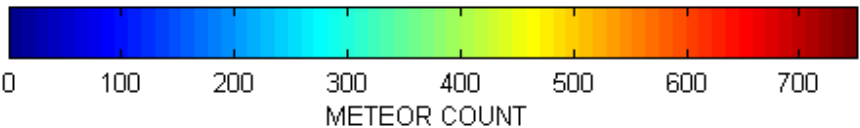

Fig. 5. The radiant distribution of all meteors observed at Ascension Island in the interval January 2005 to December 2006. All figures are plotted in the Ecliptic coordinate frame to avoid longitudinal motion of the source regions throughout the year. Contours are based upon meteor counts in $4^{\circ}$ by $4^{\circ}$ latitude/longitude bins.

Figure 5 shows that there is considerable month-to-month variability in the radiant distributions (i.e. the amplitude and the centre location of the source regions are not constant). The most conspicuous variations are (1) the very weak intensity of the northern apex source during February to April, which is mirrored by the very weak intensity of the southern apex source during September to November (2) the intensity of the northern apex source is strong in June to August and peaks in July (3) the intensity of the helion source peaks in June and the anti-helion source peaks in July (4) the helion source is below the ecliptic plane in the winter months (November to February) and above the ecliptic plane in the period late-spring to mid-autumn (May to September). The anti-helion source mirrors this behaviour and is above the ecliptic in the winter (December to February) and below the ecliptic plane in the period April to August and (5) the intensity of the northern prograde apex source has a maxima in April to July, with a shorter, secondary peak in January, and the southern prograde apex source maximises in August, November and December. 
(a)

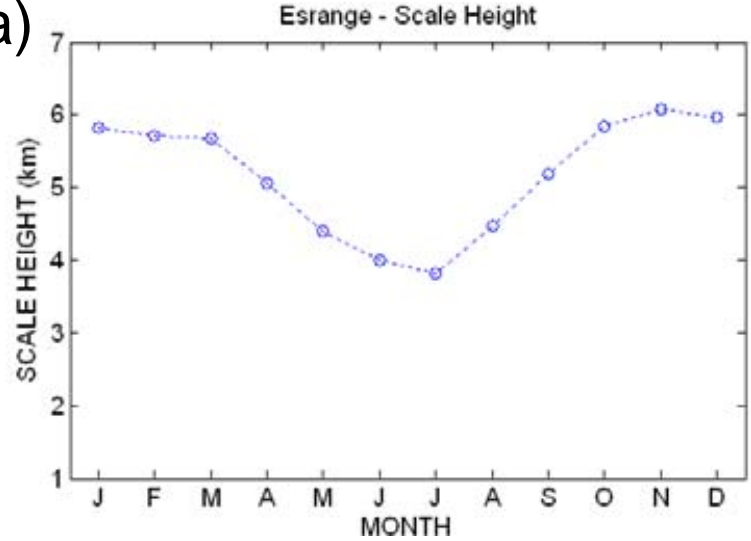

(c)

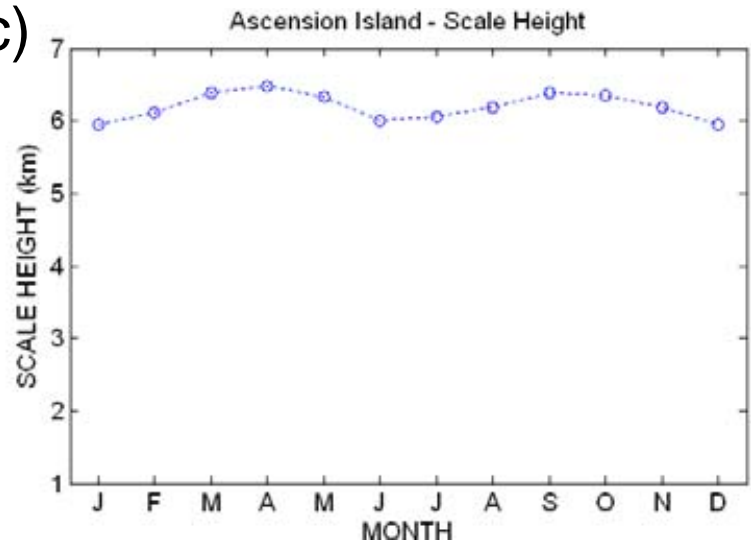

(e)

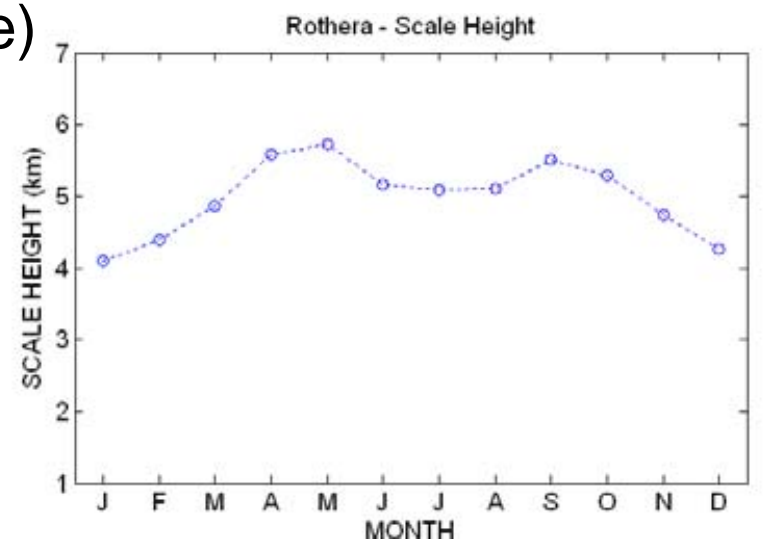

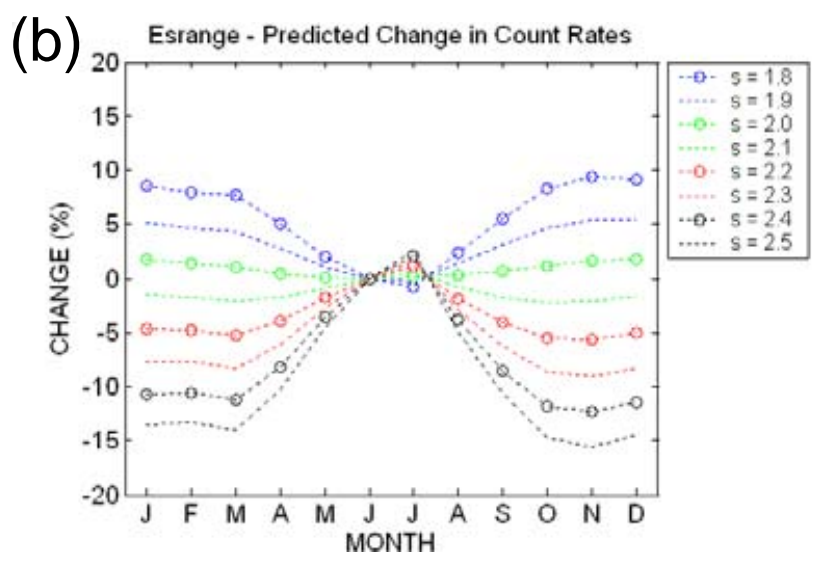

(d) Ascension Island - Predicted Change in Count Rates

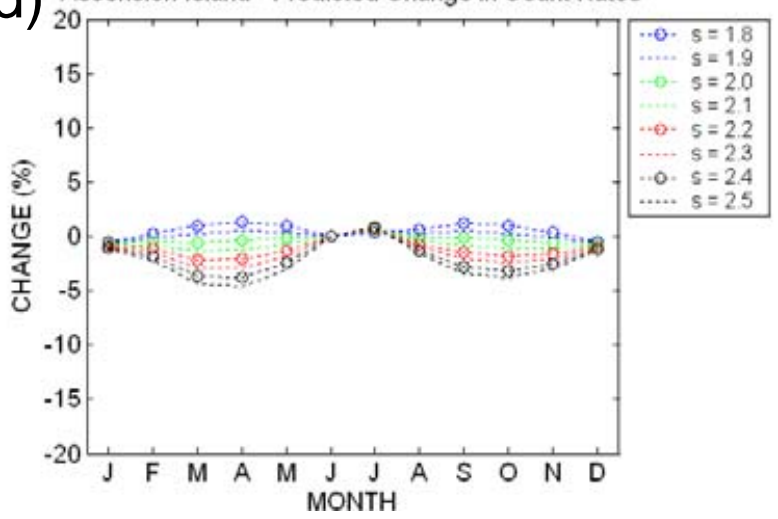

(f)

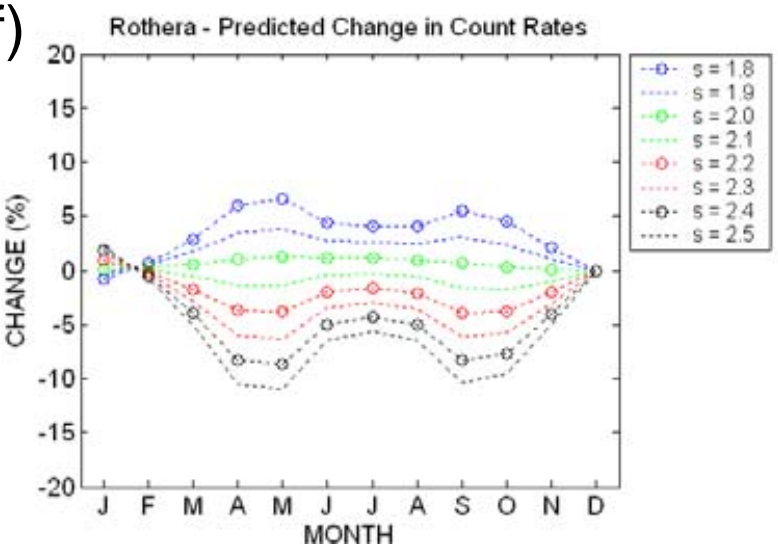

Fig. 6. (a) The monthly-mean atmospheric scale height over Esrange (at $90 \mathrm{~km}$ ) from temperature and density values taken from the MSIS90 model, (b) Percentage change in meteor count rate as a function of time and meteor mass index $s$. Change is expressed as percentage deviation away from maximum values, (c) As Fig. 5a but for Ascension Island. (d) As Fig. 5b but for Ascension Island, (e) As Fig. 5a but for Rothera and (f) As Fig. 5b but for Rothera.

\section{The effect of atmospheric density perturbations}

Figure $6 \mathrm{a}$ to $\mathrm{f}$ shows both the seasonal variations in scale height from the MSIS-90 model and the expected effect these changes should have on meteor count rates at a height of $90 \mathrm{~km}$ at Esrange, Ascension Island and Rothera. At Esrange atmospheric scale heights follow an annual cycle with scale heights of $\sim 6 \mathrm{~km}$ in the winter and $\sim 4 \mathrm{~km}$ in the summer months (Fig. 6a and b). The transition from winter to summer values (and vice versa) is gradual. The changes in the meteor count rate associated with these changes in atmospheric scale height are calculated following Ellyett and Kennewell (1980). The results of these calculations are shown in Fig. $6 \mathrm{~b}$ (as \% change relative to the maximum value) as 
function of the mass index s of observed meteors. This shows that the effects that changes in atmospheric density have on meteor count rate is strongly dependent upon $s$. By assuming that fluctuations in count rates only depend upon atmospheric density fluctuations it is expected that maximum count rates should occur in July. This is in agreement with the time of the maximum observed meteor count rate (Fig. 2). However, the shape and amplitude of the observed meteor count rate distribution is not well reproduced (see, for example, Fig. 2 and Singer et al., 2004).

At Rothera atmospheric scale heights are $\sim 5 \mathrm{~km}$ in the winter months, $\sim 5.5 \mathrm{~km}$ at the equinoxes and $\sim 4 \mathrm{~km}$ in the summer months (Fig. 6e and f). Again, following Ellyett and Kennewell (1980), the associated changes in count rate are calculated. This is displayed in Fig. 6f. From this figure, we find that maximum count rates are expected to occur in January and minimum count rates should occur in May and September. A secondary maximum in the interval June to July is also predicted. The time of maximum and minimum count rates (January and September, respectively) agrees well with observations (see Fig. 2). However, a minimum at the autumnal equinox is not observed and the observed amplitude of the annual variation in count rates is a factor of four larger than that predicted by the method of Ellyett and Kennewell (1980).

At Ascension Island a semi-annual cycle in scale height implies that a semi-annual cycle in observed meteor count rate is predicted (Fig. 6c and d). This semi-annual cycle should have a maximum in January and July. The timing of these maxima is in agreement with observations. The transition between solstical maxima and equinoctial minima also agrees with observations. However, the expected variation in meteor count rates calculated following the method of Ellyett and Kennewell (1980) (of 3\%) does not match the $30 \%$ variations in count rate observed over Ascension Island (Fig. 2).

Hence, the work presented here suggests that either the annual variation in MSIS scale height at the three locations is insufficient to account for the observed change in count rate or unrealistic values for the mass index s must be assumed to bring in to agreement the observed and theoretical variations in count rate.

\section{Conclusions}

Simultaneous observations of meteor count rates using two identical VHF radars at Arctic and Antarctic latitudes show large annual, and inter-annual, fluctuations. However, in both there are maxima in observed count rate in their respective summer season and a minimum near their spring equinox. That is, the time of maximum (or minimum) in count is out of phase by six months between the radar in the Northern Hemisphere and the radar in the Southern Hemisphere. Thus, the seasonal variation in the number of observed meteors cannot

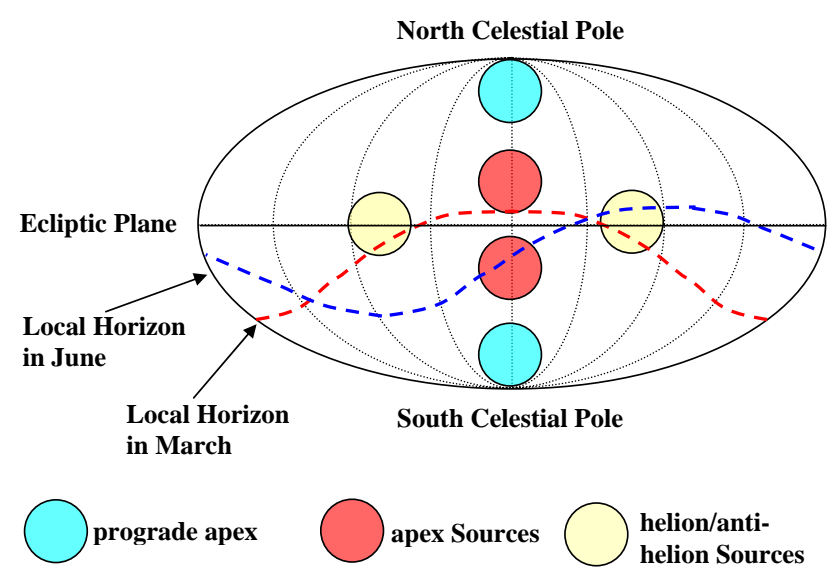

Fig. 7. Schematic of the sporadic sources in the Ecliptic coordinate frame. The helion and anti-helion sources are coloured yellow, The northern and southern apex sources are red and the prograde apex sources are coloured blue. The local horizon at Esrange in March is denoted by the red dotted line, and the local horizon in June-July is denoted by the blue dotted line.

be due to an asymmetric solar system meteoroid dust cloud as this would cause maxima to occur simultaneously in both hemispheres. In contrast, a semi-annual variation in meteor count rate is observed using a similar radar at Ascension Island (near the Equator). In this radar the observed meteor count rate has maxima in both summer and winter and minima in spring and autumn. One possibility is that the seasonal fluctuations are due to seasonal fluctuations in atmospheric scale-height. However, by modelling this it is found that although the fluctuations in scale height produces maxima and minima at similar times to those observed at all three radars the magnitude of the modelled fluctuations is too small (by a factor of 4 to 10) to account for the observed variation. Thus, another mechanism must also be acting to cause the observed seasonal variability in meteor echo count rates. One possibility is the motion of the Earth.

The annual rotation of Earth around the Sun will causes the elevation angle of the sporadic sources to move up and down through the year like the Constellations. As discussed in Sect. 2, this change in elevation will change the sensitivity of the radars to meteors from each source. Hence, even if the meteor flux was constant one would expect there to be a seasonal variation in observed count rate at each radar due to seasonal changes in elevation. The daily rotation of the Earth about its own axis also causes the sporadic sources to rise and set during the course of the day. This will cause the number of meteors observed to vary throughout the day depending on which sources are in the view of the radar. Hence, the motion of the Earth around the Sun and about its own axis will cause both a seasonal variation in the total daily meteor count rate and a change in the diurnal cycle in hourly count rate. To investigate this further the location of the sporadic sources 
(a)

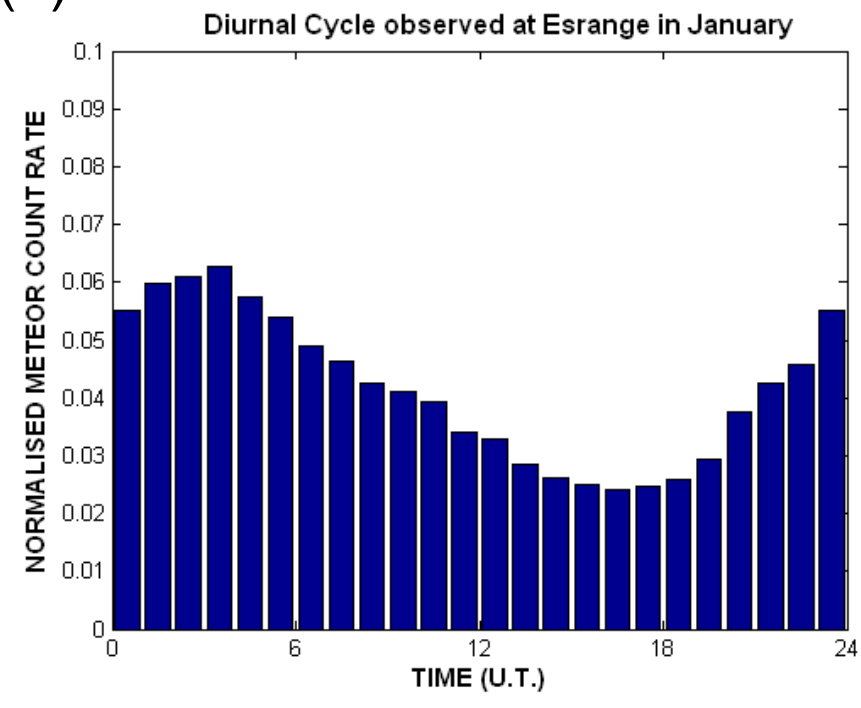

(b)

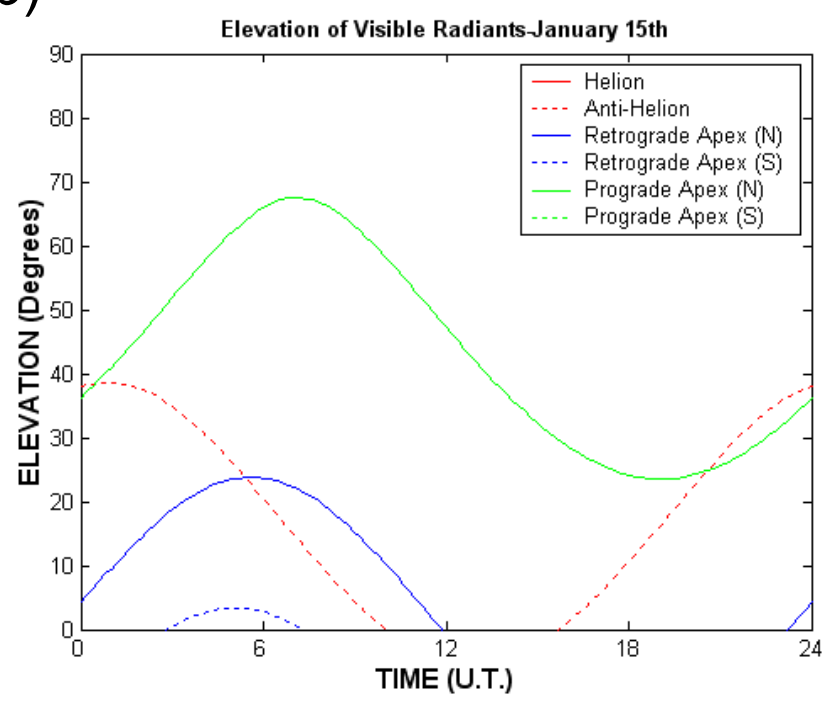

(c)

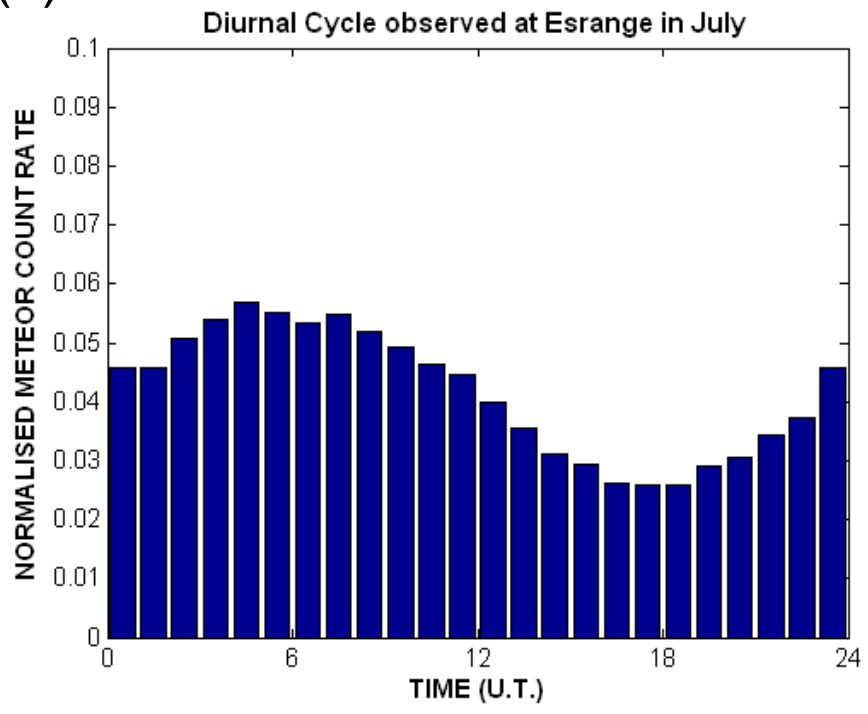

(d)

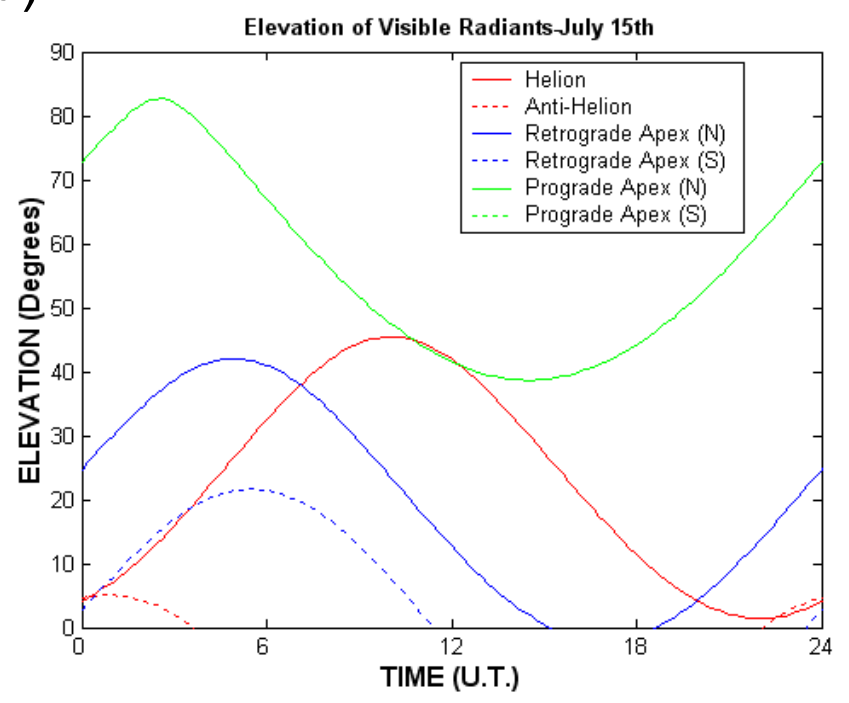

Fig. 8. Hourly meteor count rate observed at Esrange on (a) January 2004 and (b) July 2004 (Note the use of universal time). The elevation angles of the six source regions of the sporadic radiant distribution are shown in (a) for 15 January 2004 and (b) for 15 July 2004.

and the field of view of the Esrange radar were determined. These are displayed in Fig. 7 for March (red dotted line) and June/July (blue dotted line). These two periods correspond to the time of minimum and maximum meteor count rates respectively. In March the southern apex and southern prograde apex source regions are below the local horizon. Hence meteors from these source regions can not be observed at Esrange. The northern apex, helion and anti-helion sources are also close to the local horizon (within $\sim 20^{\circ}$ ) thus lessening the sensitivity of the radar to these sources (Sect. 2). This poor viewing geometry appears responsible for the low meteor count rates observed in March. However, in June and July the helion, anti-helion, southern apex and northern sources are all above the local horizon and thus all contribute to observed count rates. As discussed in Sect. 4, and above, at this time the change in density structure of the atmosphere also results in the detection of fainter meteors than at any other time of the year. Thus, the combination of good viewing geometry and the atmospheric conditions results in the large count rates observed in the summer months. Similar arguments can be used to explain the count rate distributions at Rothera and Ascension Island. 
Similarly, the variation in the local time of the peak in meteor count rate with season and geographic latitude can also be explained by the change in elevation angle of each sporadic meteor radiant with season and latitude. In particular, at high latitudes the elevation angle of a meteor source close to the ecliptic plane at a particular hour depends strongly on the time of year. However, at low latitudes the elevation angle of a source close to the ecliptic plane, at a particular hour, does not vary much with time of year. Thus, since the stronger sporadic sources are close to the ecliptic plane the largest deviations in the time of peak meteor count rates will occur at high latitudes. This effect is demonstrated in Fig. 8 a to d. Figure 8a shows the diurnal cycle in meteor count rates observed at Esrange in January 2004. Above average count rates are observed between 00:00 and 07:00 UT and between 23:00 and 24:00 UT, and below average count rates are observed between 07:00 and 23:00 UT. Figure 8b shows the elevation angle of the sporadic sources for an observer at Esrange in mid-January 2004. At times of high count rates the anti-helion and northern prograde apex sources have elevation angles between $25^{\circ}$ and $70^{\circ}$. Hence, the observed maximum is, at least in part, due to the increase in sensitivity of the radar to sources at these elevations. The morning enhancement in count rates is also, in part, increased by an increased sensitivity of the radar to the apex sources. Similarly, the smallest count rates are observed $(\sim 17: 00 \mathrm{UT})$ when all of the sporadic sources are either below or close to the local horizon. Figure $8 \mathrm{c}$ and $\mathrm{d}$ shows the diurnal cycle in meteor count rates observed at Esrange in July 2004 and the corresponding elevations of the sporadic sources. In this case, the diurnal cycle in count rates has a broader and later morning enhancement (between 02:00 and 12:00 UT) than in January. This broader enhancement is caused by the later passage of the helion source across the sky.

Thus, in addition to the background temporal and spatial variability in meteor flux, the latitudinal, spatial and temporal dependence in observed meteor count rates appear to result from a combination of the sporadic radiant distribution, annual fluctuations in atmospheric density and the sensitivity of the radar to meteors from different source elevations.

Acknowledgements. The authors wish to thank Jules, Mark, Neil, and all of the other personnel at Rothera research station for all of their hard work.

Topical Editor C. Jacobi thanks M. Campbell-Brown and another anonymous referee for their help in evaluating this paper.

\section{References}

Baggaley, W. J.: Radar observations, in: Meteors in the Earth's Atmosphere, Cambridge Univ. Press, 123-148, 2002.

Brown, P. and Jones, J.: A determination of the strength of the sporadic radio meteor sources, Earth, Moon Planets, 68, 223-245, 1995.

Campbell-Brown, M. D. and Jones, J.: Annual variations of sporadic radar meteor rates, Mon. Nat. R. Astron. Soc., 367(2), 709716, doi:10.11111/j.1365-2966.205.09974.x, 2006.

Cervera, M. A. and Elford, W. G.: The meteor radar response function: Theory and application to narrow beam MST radar, Planet. Space Sci., 52, 591-602, 2004.

Ceplecha, Z., Borovicka, J., Elford, W. G., Revelle, D. O., Hawkes, R. L., Porubcan, V., and Simek, M.: Meteor phenomena and bodies, Space Sci. Rev., 84, 327-471, 1998.

Ellyett, C. D. and Kennewell, J. A.: Radar meteor rates and atmospheric density changes, Nature, 287, 521-522, 1980.

Galligan, D. P. and Baggaley, W. J.: The orbital distribution of radar-detected meteors of the solar system dust cloud, Mon. Not. R. Astron. Soc., 353, 422-449, 2004.

Galligan, D. P. and Baggaley, W. J.: The radiant distribution of AMOR radar meteors, Mon. Not. R. Astron. Soc., 359, 551-560, 2005

Hawkins, G. S.: A radio echo survey of sporadic meteor radiants, Mon. Not. R. Astron. Soc., 116, 92-104, 1956.

Hocking, W. K., Fuller, B., and Vandepeer, B.: Real-time determination of meteor-related parameters utilizing modern digital technology, J. Atmos. Solar. Terr. Phys., 63, 155-169, 2001.

Jones, J. and Brown, P.: Sporadic meteor radiant distribution: Orbital survey results, Mon. Not. R. Astron. Soc., 265, 524-532, 1993.

Jones, J. and Campbell-Brown, M.: The initial train radius of sporadic meteors, Mon. Not. R. Astron. Soc., 359, 1131-1136, 2005.

Kaiser, T. R. and Closs, R. L.: Theory of radio reflections from meteor trails .1., Phil. Mag., 43, 1-32, 1952.

Kaiser, T. R.: The determination of the incident flux of radiometeors, Mon. Not. R. Astr. Soc., 121, 284-298, 1960.

Kaiser, T. R.: The determination of the incident flux of radiometeors .2. Sporadic meteors, Mon. Not. R. Astr. Soc., 123, 265271, 1961.

McKinley, D. W. R.: Meteor Science and Engineering, McGrawHill, 1961.

Simek, M. and McIntosh, B. A.: Meteor mass distribution from underdense trail echoes, in: Physics and Dynamics of Meteors, Riedel Publishing Company, 362-372, 1968.

Singer, W., von Zahn, U., and Weiß, J.: Diurnal and annual variations of meteor rates at the arctic circle, Atmos. Chem. Phys., 4, 1355-1363, 2004 http://www.atmos-chem-phys.net/4/1355/2004/.

Taylor, A. D. and Elford, W. G.: Meteoroid orbital element distributions at $1 \mathrm{AU}$ deduced from the Harvard Radio Meteor Project, Earth Planets Space, 50, 569-575, 1998. 